\title{
Fatty acid synthetase expression in triple-negative breast cancer
}

\author{
Jin Hee Park ${ }^{1}$, Hye Seung Han ${ }^{1}$, So Dug Lim¹, Wook Youn Kim ${ }^{1}$, Kyoung Sik Park ${ }^{2}$, \\ Young Bum Yoo ${ }^{2}$, Seung Eun Lee', Wan-Seop Kim ${ }^{1}$ \\ Departments of ${ }^{1}$ Pathology and ${ }^{2}$ Surgery, Konkuk University Medical Center, Konkuk University School of Medicine, Seoul, Korea
}

\begin{abstract}
Background: Triple-negative breast cancer (TNBC) has a relatively poor prognosis. Research has identified potential metabolic targets, including fatty acid metabolism, in TNBC. The absence of effective target therapies for TNBC led to exploration of the role of fatty acid synthetase (FASN) as a potential target for TNBC therapy. Here, we analyzed the expression of FASN, a representative lipid metabolismrelated protein, and investigated the association between FASN expression and Ki-67 and the programmed death ligand 1 (PD-L1) biomarkers in TNBC. Methods: Immunohistochemical expression of FASN was analyzed in 166 patients with TNBC. For analytical purposes, patients with 0-1+ FASN staining were grouped as low-grade FASN and patients with 2-3+ FASN staining as high-grade FASN. Results: FASN expression was observed in $47.1 \%$ of TNBC patients. Low and high expression of FASN was identified in $75.9 \%$ and $24.1 \%$, respectively, and no statistically significant difference was found in T category, $\mathrm{N}$ category, American Joint Committee on Cancer stage, or recurrence rate between the low and high-FASN expression groups. Ki-67 proliferation level was significantly different between the low and high-FASN expression groups. FASN expression was significantly related to Ki-67 as the level increased. There was no significant difference in PD-L1 positivity between the low- and high-FASN expression groups. Conclusions: We identified FASN expression in 166 TNBC patients. The Ki-67 proliferation index was positively correlated with FASN level, indicating higher proliferation activity as FASN increases. However, there was no statistical association with PD-L1 SP142, the currently FDA-approved assay, or FASN expression level.
\end{abstract}

Key Words: Triple-negative breast neoplasms; FASN; Immunohistochemistry; Ki-67; PD-L1 SP142

Received: September 29, 2021 Revised: October 20, 2021 Accepted: October 27, 2021

Corresponding Author: Seung Eun Lee, MD, PhD, Department of Pathology, Konkuk University Medical Center, Konkuk University School of Medicine, 120-1 Neungdong-ro, Gwangjin-gu, Seoul 05030, Korea

Tel: +82-2-2030-5644, Fax: +82-2-2030-5629, E-mail: 20150063@kuh.ac.kr

Corresponding Author: Wan-Seop Kim, MD, PhD, Department of Pathology, Konkuk University Medical Center, Konkuk University School of Medicine, 120-1 Neungdong-ro, Gwangjin-gu, Seoul 05030, Korea

Tel: +82-2-2030-5642, Fax: +82-2-2030-5629, E-mail: wskim@kuh.ac.kr

Triple-negative breast cancer (TNBC) is defined by a loss of expression of estrogen receptor (ER), progesterone receptor (PR), and human epidermal growth factor receptor 2 (HER-2) [1]. TNBC accounts for about $15 \%$ of all breast cancers [2]. TNBC has relatively poor prognosis compared to non-TNBC patients, resulting from a lack of specific treatment methods such as hormone treatment or targeted treatment other than non-specific chemotherapy [3]. TNBC is a heterogeneous group of tumors with different molecular drivers and prognosis, clinical outcomes, and responses to therapy [4]. Various inhibitors, including poly (ADP-ribose) polymerase (PARP), growth factor, Scr, mammalian target of rapamycin (mTOR), and phosphoinositide 3-kinase (PI3K) inhibitors, are intended to increase treatment effectiveness [5]. Based on the high level of programmed death ligand 1 (PD-
L1) expression in TNBC, chemotherapy such as atezolizumab and nab-paclitaxel has been approved by the Food and Drug Administration (FDA) as the first-line treatment in breast cancer patients with locally advanced and metastatic TNBC [6]. Atezolizumab plus nab-paclitaxel-treated patients are linked to improved clinical outcome [7].

The study of metabolic pathways in TNBC is also active, and studies [8] have shown that potential metabolic targets of TNBC include glucose metabolism, fatty acid metabolism, glutamine metabolism, and serine metabolism. Gong et al. [9] identified three metabolic pathway-based TNBC subtypes with distinct molecular features and sensitivities to various metabolic inhibitors, and it was shown that inhibition of lactate dehydrogenase could enhance the anti-programmed death-1 immunotherapy 
response in a certain TNBC subtype. Cha et al. [10] identified lipid metabolism-related proteins in breast cancer. Of them, fatty acid synthetase (FASN) is a lipid-producing enzyme that is expressed at low level in normal human tissues but is reported to be highly expressed in breast, colon, prostate, and ovarian cancer [11-14]. The role of FASN as a prognostic factor in breast cancer has been published in previous studies $[15,16]$.

However, few studies have identified the expression of FASN level in TNBC $[15,16]$. The absence of effective targeted therapy for TNBC and its poor prognosis led to exploration of the role of FASN as a potential target for TNBC therapy [17]. Here, we analyzed the expression of FASN, a representative lipid metabolism-related protein, and the association between FASN level and Ki-67 and PD-L1 expression in patients with aggressive TNBC breast cancer.

\section{MATERIALS AND METHODS}

Among ER-and HER-2-negative breast cancer patients, 166 patients who could be tracked and whose paraffin embedded tissue blocks were in good condition were enrolled in the study. Patient clinical records were used in a retrospective manner to identify clinical information including age, immunohistochemical study, TNM stage, recurrence, chemotherapy, and radiation therapy. We also wanted to quantify the degree of expression and correlate it with clinical information through immunostaining of FASN.

\section{Patient selection and clinicopathologic evaluation}

From January 2012 to December 2018, 166 TNBC patients who underwent primary breast cancer surgical resection at Konkuk University Medical Center (KUMC) Seoul, Korea, were analyzed. Clinicopathological information was obtained by reviewing medical records and hematoxylin and eosin (H\&E)-stained sections. The TNBC histopathological data were determined by histological subtype, category T, category N, American Joint Committee on Cancer (AJCC) stage, and Bloom-Richardson histological grade.

\section{Tissue microarray}

All 166 H\&E-stained slides were reviewed, and the most representative part of each was selected. Two $3 \mathrm{~mm}$ tissue cores derived from representative tumors of formalin-fixed paraffin-embedded tissue blocks were collected. An on-slide control tissue (tonsillar tissue) was used.

\section{Immunohistochemistry}

Immunohistochemistry (IHC) was performed using a Ventana Discovery XT automated slide stainer (Ventana Medical Systems, Tucson, AZ, USA), following antigen retrieval with Cell Conditioning 1 (CC1; citrate buffer $\mathrm{pH}$ 6.0, Ventana Medical System) at $37^{\circ} \mathrm{C}$ for 32 minutes. This was followed by a standard Ventana signal amplification and counterstaining with hematoxylin. Slides were mounted and examined by light microscopy. Appropriate positive and negative controls for IHC were included. We used anti-fatty acid synthase antibodies (Abcam, Cambridge, UK) and SP142 antibodies (Ventana, Mannheim, Germany) in this study.

\section{Interpretation of $\mathrm{IHC}$ results}

All IHC markers were determined using light microscopy to assess the proportion and intensity of stained cells. In this study, we classified breast cancer phenotypes according to ER, PR, and HER-2 IHC results and Ki-67 [18]. ER and PR positivity were used to define a cutoff value of $1 \%$ positively-stained nuclei [19]. HER-2 staining was analyzed according to the American Society of Clinical Oncology (ASCO)/College of American Pathologists guidelines [20]. HER-2 expression was considered positive when strong $(3+)$ membranous staining was observed, whereas cases with scores of 0 to $1+$ were considered negative [10]. The results of immunohistochemical staining for lipid metabolism-related proteins were scored as previously described [21]. Briefly, FASN staining was considered positive when $>10 \%$ of the tumor cells were stained, and the intensity was scored from 0 to 3 . For analytical purposes, patients with $0-1+$ FASN staining were grouped as low-grade FASN and patients with 2-3+ FASN staining as high-grade FASN [15]. SP142 score was assessed as previously described [6].

\section{Statistical analysis}

Data analysis was performed using SPSS ver. 20.0 (IBM Corp., Armonk, NY, USA) for Windows. Chi-square and Fisher's exact tests were used to assess continuous and categorical variables, respectively. Statistical significance was assumed at a p-value $<.05$. Kaplan-Meier survival curves and log-rank statistics were employed to evaluate the time interval to tumor metastasis and survival duration. A Cox proportional hazards model was used to assess the risk factors of shorter disease-free survival and overall survival. 


\section{RESULTS}

\section{Clinicopathological characteristics of TNBC}

A total of 166 TNBC patient results were analyzed in this study, and the clinicopathological characteristics are presented in Table 1 . The median age at diagnosis was 51 years (range, 28 to 83

Table 1. Clinicopathological characteristics of 166 patients with TNBC

\begin{tabular}{|c|c|}
\hline Characteristics & No. of patients \\
\hline Age (yr) & $51(28-83)$ \\
\hline \multicolumn{2}{|l|}{ Age (yr) } \\
\hline$\leq 50$ & $81(48.8)$ \\
\hline$>50$ & $85(51.2)$ \\
\hline \multicolumn{2}{|l|}{ Histologic type } \\
\hline IDC, NOS & $154(92.8)$ \\
\hline Carcinoma with medullary feature & $8(4.8)$ \\
\hline Pleomorphic carcinoma & $3(1.8)$ \\
\hline Metaplastic carcinoma & $1(0.6)$ \\
\hline \multicolumn{2}{|l|}{ T category } \\
\hline 1 & $66(39.8)$ \\
\hline 2 & $88(53.0)$ \\
\hline 3 & $11(6.6)$ \\
\hline 4 & $1(0.6)$ \\
\hline \multicolumn{2}{|l|}{ N category } \\
\hline 0 & $109(65.7)$ \\
\hline 1 & $40(24.1)$ \\
\hline 2 & $9(5.4)$ \\
\hline 3 & $4(2.4)$ \\
\hline NA & $4(2.4)$ \\
\hline \multicolumn{2}{|l|}{ Histologic grade } \\
\hline 1 & 0 \\
\hline 2 & $24(14.5)$ \\
\hline 3 & $142(85.5)$ \\
\hline \multicolumn{2}{|l|}{ AJCC stage } \\
\hline 1 & 33 (19.9) \\
\hline 2 & $110(66.3)$ \\
\hline 3 & $19(11.4)$ \\
\hline $\mathrm{N} / \mathrm{A}$ & $4(2.4)$ \\
\hline \multicolumn{2}{|l|}{ Neoadjuvant CTx } \\
\hline Yes & $10(6.0)$ \\
\hline No & $156(94.0)$ \\
\hline \multicolumn{2}{|l|}{ CTx } \\
\hline Yes & $154(92.8)$ \\
\hline No & $12(7.2)$ \\
\hline \multicolumn{2}{|l|}{$\mathrm{RT} x$} \\
\hline Yes & $140(84.3)$ \\
\hline No & $26(15.7)$ \\
\hline \multicolumn{2}{|l|}{ Recur } \\
\hline Yes & $26(15.7)$ \\
\hline No & 140 (84.3) \\
\hline
\end{tabular}

Values are presented as median (interquartile range) or number (\%). TNBC, triple-negative breast cancer; IDC, invasive ductal carcinoma; NOS, not otherwise specified; NA, not applicable; AJCC, American Joint Committee on Cancer; CTx, chemotherapy; RTx, radiation therapy. years). The major histologic type of TNBC in KUMC was invasive ductal carcinoma (92.8\%), with eight carcinomas with medullary features, three pleomorphic carcinomas, and one metaplastic carcinoma. About $85 \%$ of the cases were histologic grade 3, and $66 \%$ of the cases were AJCC stage 2. Of these 166 patients, 10 (6.0\%) received neoadjuvant chemotherapy, 154 (92.8\%) received adjuvant chemotherapy, and $140(84.3 \%)$ received radiation therapy. Among the 166 patients, 26 (15.7\%) recurred during follow-up observation. The median time until recurrence was 13.5 months.

\section{Expression of FASN in TNBC}

Two pathologists independently observed and agreed to the final score values, and in some cases, IHC study was performed up to three times. The FASN expression levels in breast cancer patients were classified as grade $0,1,2$, or 3 according to staining intensity. FASN expression was observed in 47.1\% (95/166) of TNBC patients. Intensity grades of 1,2 , or 3 were seen in $33.1 \%$ (55/166), $13.3 \%$ (22/166), and 10.8\% (18/166) of TNBC patients, respectively. Grades 0 and 1 were classified as the lowFASN group and grades 2 and 3 as the high-FASN group (Fig. 1). Low and high expression of FASN was identified in $75.9 \%$ (126/166) and 24.1\% (40/166) of TNBC patients, respectively. Table 2 shows a comparison between the clinicopathological data of 40 patients with high FASN expression compared with that of 126 patients with low FASN expression. In the high-FASN expression group, more patients were younger than 50 years, while in the low-FASN group, more patients were older than 50 years old. The major cancer type was invasive ductal carcinoma and most were histologic grade 3; therefore, there was no association with high and low FASN expression. No statistically significant differences in T category, $\mathrm{N}$ category, AJCC stage, and recurrence rate were found between the high- and low-FASN expression groups.

\section{Correlation with immunohistochemical study of Ki-67}

The Ki-67 proliferation level was statistically different between the FASN low and high expression groups, with median Ki-67 levels of $60 \%$ (range, $40 \%$ to $80 \%$ ) and $73 \%$ (range, $55 \%$ to $87 \%)$, respectively, using the Mann-Whitney $\mathrm{U}$ test $(\mathrm{p}=.003)$ (Table 3). The Spearman correlation coefficient was 0.257, indicating weak correlation between Ki-67 proliferation level and FASN expression level ( $\mathrm{p}=.001)$.

\section{Correlation with PD-L1 SP142 IHC assay}

Two pathologists independently observed and agreed to the 

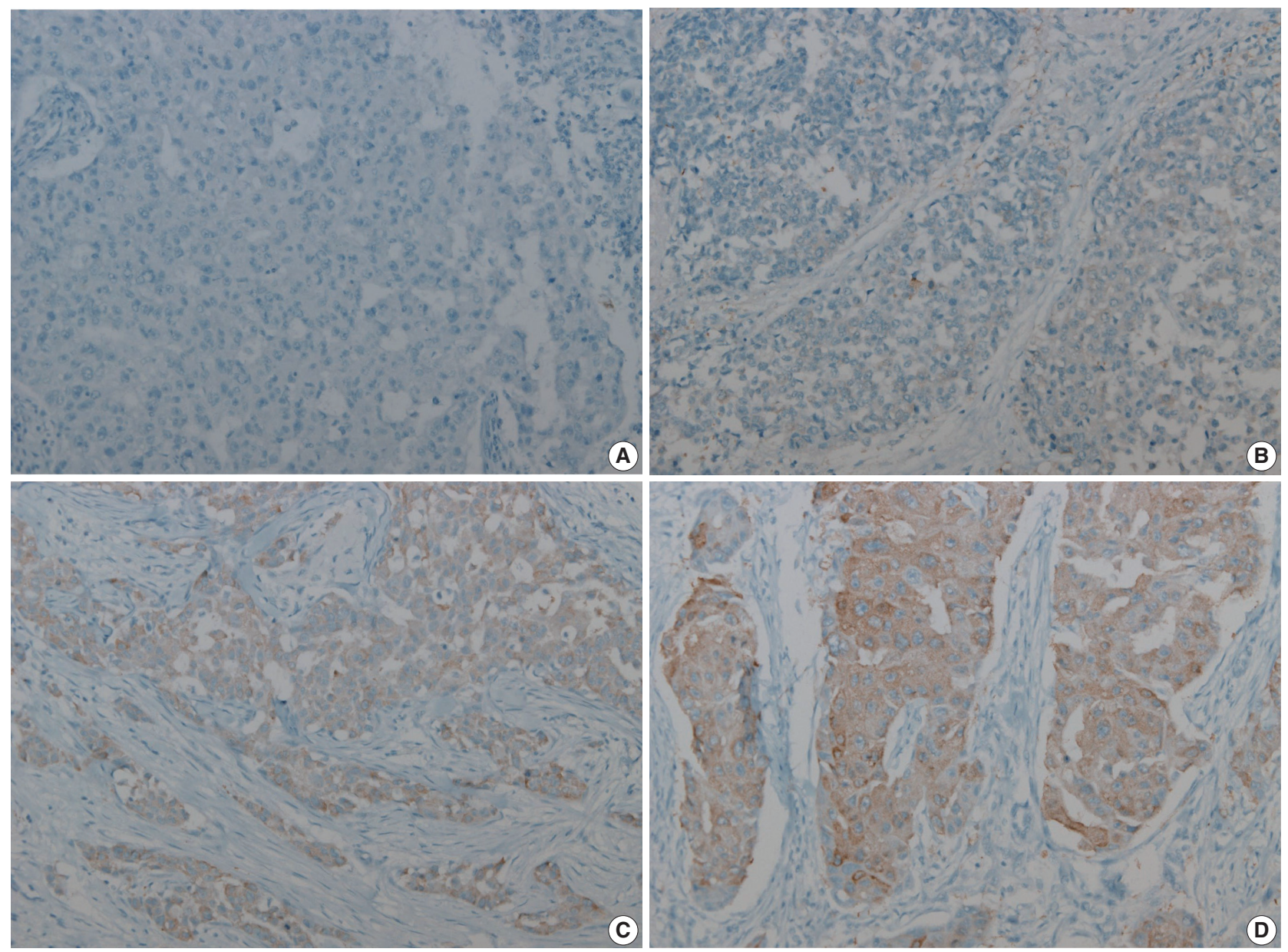

Fig. 1. Fatty acid synthetase expression in triple-negative breast cancer: grade $0(A)$, grade $1(B)$, grade $2(C)$, and grade 3 (D).

final score values. PD-L1 expression was assessed using the PDL1 SP142 assay. Of the 166 cases, 94 available cases were used to perform IHC for PD-L1 SP142. At the 1\% cutoff value, $52.6 \%$ (50/94) of cases were positive for PD-L1 SP142 in infiltrating immune cells (ICs). We analyzed the correlation between FASN expression level and percentage of PD-L1 SP142 positive infiltrating ICs, but no correlation was identified. There was no statistically significant PD-L1 SP142 positivity between the low- and high-FASN expression groups. A representative case of SP142 expression in the expression of high FASN was confirmed (Fig. 2).

\section{DISCUSSION}

TNBC has no available special treatment other than chemotherapy after surgery, so early detection of recurrence through short-term follow-up observations will help patients with treatment and prognosis [22]. The development of interval breast cancer within the time interval between screening examinations has more adverse biological features, poorer survival outcomes, and is more highly associated with TNBC [23]. TNBC has high prevalence in young women under 50 and is more common in African Americans [24]. TNBC is known to have relatively poor prognosis compared to non-triple-negative breast cancer, most likely resulting from lack of special treatment methods such as hormone treatment or targeted treatment other than non-specific chemotherapy [25].

The TNBC subtype was classified into four distinct types by Burstein et al. [26] and is being actively studied because of different prognoses and target therapies. Clinical results and treatment responses have changed depending on classification [27]. These inherent differences related to TNBC sub-classification have resulted in a renewed effort to identify driver mutations and more appropriate targeted treatment [28]. For potential therapy, activated progesterone receptor, platinum, tyrosine kinase inhibitor, PI3K/mTOR inhibitor, anti-PD-L1 inhibitors, and/or androgen receptor targeted $\mathrm{PI} 3 \mathrm{~K}$ inhibitors are thought to be 
Table 2. Clinicopathological data for 40 patients with high-grade FASN compared with 126 patients with low-grade FASN

\begin{tabular}{|c|c|c|c|}
\hline & Low $(n=126)$ & High $(n=40)$ & $p$-value \\
\hline Age (yr) & & & $.018^{a}$ \\
\hline$<50$ & $58(46.0)$ & $27(67.5)$ & \\
\hline$>50$ & $68(54.0)$ & $13(32.5)$ & \\
\hline Histologic type & & & $.319^{b}$ \\
\hline IDC, NOS & $116(92.1)$ & 38 (95.0) & \\
\hline Carcinoma with medullary feature & $7(5.6)$ & $1(2.5)$ & \\
\hline Pleomorphic carcinoma & $3(2.4)$ & 0 & \\
\hline Metaplastic carcinoma & 0 & $1(2.5)$ & \\
\hline T category & & & $.080^{\mathrm{b}}$ \\
\hline 1 & $44(66.7)$ & $22(33.3)$ & \\
\hline 2 & $73(83.0)$ & $15(17.0)$ & \\
\hline 3 & $8(72.7)$ & $3(27.3)$ & \\
\hline 4 & $1(100)$ & 0 & \\
\hline $\mathrm{N}$ category & & & $.614^{b}$ \\
\hline 0 & 79 (72.5) & $30(27.5)$ & \\
\hline 1 & $33(82.5)$ & $7(17.5)$ & \\
\hline 2 & $8(88.9)$ & $1(11.1)$ & \\
\hline 3 & $2(50.0)$ & $2(50.0)$ & \\
\hline AJCC stage & & & $.075^{\mathrm{b}}$ \\
\hline 1 & $18(54.5)$ & $15(45.5)$ & \\
\hline 2 & $91(82.7)$ & $19(17.3)$ & \\
\hline 3 & $13(68.4)$ & $6(31.6)$ & \\
\hline$N / A$ & $4(100)$ & 0 & \\
\hline Histologic grade & & & $.097^{\mathrm{a}}$ \\
\hline 2 & $15(11.9)$ & $9(22.5)$ & \\
\hline 3 & $111(88.1)$ & $31(77.5)$ & \\
\hline Neoadjuvant CTx & $10(7.94)$ & 0 & $.120^{\mathrm{b}}$ \\
\hline $\mathrm{CTx}$ & $119(94.4)$ & 35 (87.5) & $.140^{\mathrm{a}}$ \\
\hline $\mathrm{RT} x$ & $105(83.3)$ & 35 (87.5) & $.528^{a}$ \\
\hline Recur & 23 (18.3) & $3(7.5)$ & $.135^{\mathrm{b}}$ \\
\hline
\end{tabular}

FASN, fatty acid synthetase; IDC, invasive ductal carcinoma; NOS, not otherwise specified; N/A, not applicable; AJCC, American Joint Committee on Cancer; CTx, chemotherapy; RTx, radiation therapy.

aLinear by linear association; 'Fisher's exact test.

possible [29]. Therefore, these treatments are being attempted in TNBC patients to identify whether they can benefit from a more appropriate and targeted treatment. We performed IHC for FASN, a representative lipid metabolism-related protein, as a candidate for a potential specific target in TNBC.

FASN is a key lipogenic enzyme and is known to be overexpressed in various human cancers [30]. FASN is a lipid-producing enzyme that is expressed at low levels in normal human tissues but is reported to be highly expressed in breast, colon, prostate, ovarian, and endometrial cancers [11-14]. It has been hypothesized that useful indicators of prognosis can be found based on differences in the degree of expression between normal and cancer tissues, and patients with high FASN expression level have been reported to show lower disease-free survival and decreased survival rate [31]. High FASN expression was identified in 45\%
Table 3. Mann-Whitney $U$ and Spearman's correlation test results with immunohistochemical Ki-67 proliferation index in low- and high-FASN groups

\begin{tabular}{lcc}
\hline Mann-Whitney U test & Ki-67 & p-value \\
\hline Low FASN, median (range) & $60(40-80)$ & .003 \\
High FASN, median (range) & $72.75(55.13-87.7)$ & \\
Spearman's correlation & & \\
FASN expression level & $\rho=0.257$ & .001 \\
\hline FASN, fatty acid synthetase. & &
\end{tabular}

of TNBC cases [15] and high-FASN groups have been significantly associated with positive nodal status $[15,16]$. Therefore, FASN has emerged as a potential target, and FASN inhibitors are being evaluated in clinical trials [32]. First-generation (e.g., orlistat and cerulenin) and next-generation (TVB-3166 and TVB2640) FASN-targeting drugs have been developed [33]. Adipophilin (ADP) and FASN are two lipid metabolism-related proteins of clinicopathological relevance for IHC expression in salivary duct carcinoma. ADP-positive expression was associated with the presence of prominent nuclear pleomorphism, high $\mathrm{Ki}-67$, and poor prognosis [30].

In this study, we evaluated the association of FASN expression between clinicopathological features, including PD-L1 SP142 expression, and further evaluated its usefulness as a biomarker in TNBC. FASN expression was observed in 47.1\% (95/166) of TNBC patients in our study. Staining intensity grades of 1, 2, and 3 were found in $33.1 \%$ (55/166), $13.3 \%$ (22/166), and $10.8 \%$ (18/166) of TNBC patients, respectively. Low (grade 0,1$)$ and high (grade 2,3) expression of FASN was identified in $75.9 \%$ (126/166) and 24.1\% (40/166) of TNBC, respectively. However, we could not identify an association with lymph node stage or TNM stage [15].

$\mathrm{Ki}-67 \mathrm{IHC}$ is used as a proliferation marker in breast cancer and is a low-cost measurement method suitable for extensive use in clinical practice [34]. Higher Ki-67 level is associated with increased early recurrence and aggression, resulting in a lower patient survival rate [35]. In a Japanese study, when Ki-67 was greater than $10 \%$, it was related with a high recurrence rate and low survival rate [36]. The Breast Cancer Working Group proposed guidelines for Ki-67 assessment in breast cancer and use of this potentially important marker based on current evidence [37]. Less than $5 \%$ of patients do not require chemotherapy, and more than 30\% show chemotherapy indication. The ER negative case is referred to as insufficient evaluation for Ki-67 [37]. Another group reported that high Ki-67 expression (cutoff value of $30 \%$ ) was correlated with early recurrence in luminal B/HER-2 negative breast cancer [38]. TNBC patients in the high Ki-67 

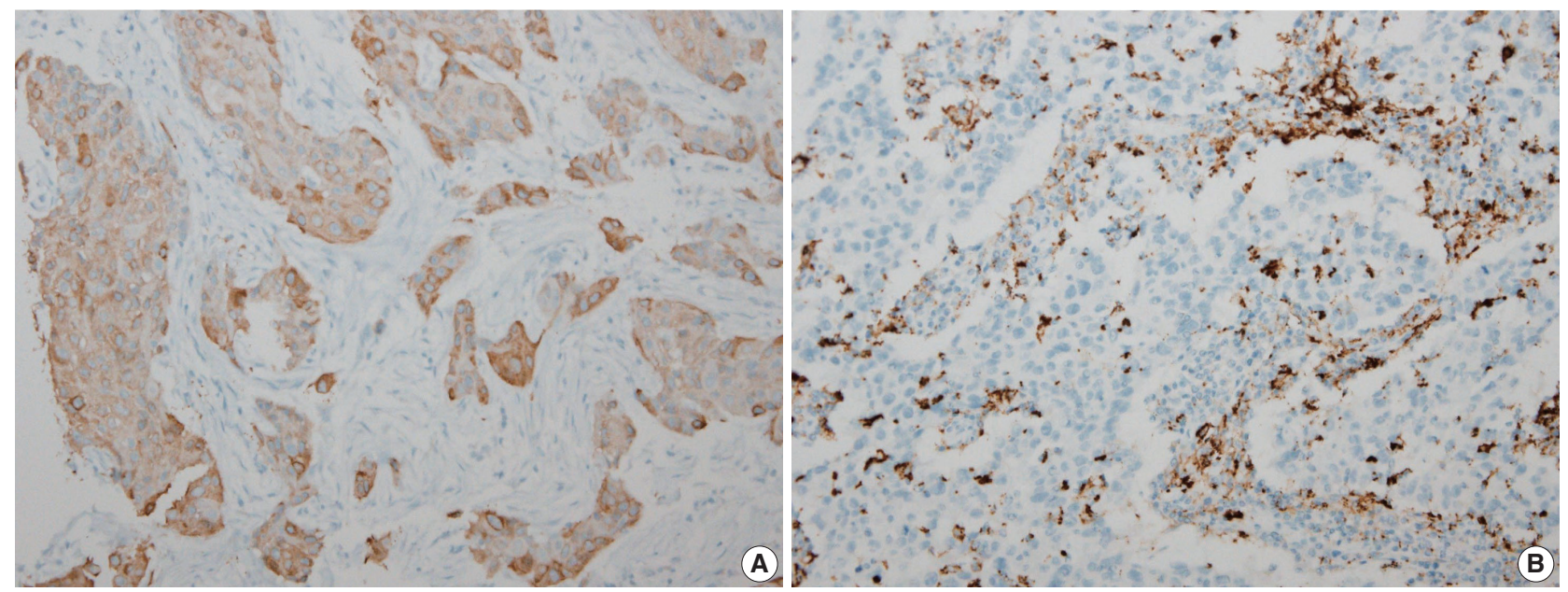

Fig. 2. A representative case of SP142 (B) expression in the expression of high fatty acid synthase (FASN) (A) was confirmed.

group seem to benefit more from treatment with carboplatin [39]. The majority of TNBC cases has high Ki-67 expression level $(\geq 20 \%)$, which is used as a proliferation factor [40]. In our study, $92.8 \%$ of cases had Ki-67 expression $\geq 20 \%$. However, the Ki-67 proliferation level was statistically different between the FASN low and high expression groups. The median Ki-67 value of the low- and high-FASN expression groups was $60 \%$ (range, $40 \%$ to $80 \%$ ) and $73 \%$ (range, $55 \%$ to $87 \%$ ), respectively.

In 2019, the FDA-approved atezolizumab (TECENTRIQ, Genentech Inc.) treatment in combination with paclitaxel was approved for TNBC patients whose PD-L1 stained tumor-infiltrating ICs were over $1 \%$ intensity [41]. PD-L1 is a cell membrane protein expressed in tumor cells and ICs [42]. PD-L1 expression is increased in TNBC and is a positive predictor of immunotherapy [43]. PD-L1 IHC using the VENTANA SP142 assay, the current FDA-approved assay, improved clinical outcome in atezolizumab plus nab-paclitaxel-treated patients [44]. In our study, we investigated the association between PD-L1 and FASN expression level and unfortunately identified no association.

TNBC represents a small proportion of breast cancer subtypes but has the worst outcome [45]. Although this study was based on data from a small number of TNBC cases and short-term follow-up surveys, we included 166 TNBC patients in the study. More research on the expression of FASN on TNBC and its potential inhibitors will help identify new target treatments. Further research is needed on how FASN correlates with other factors in TNBC. In addition, further studies are required with a larger number of TNBC patients to investigate the specific role of FASN in TNBC and the possibility for FASN inhibition as a target treatment for TNBC.

In conclusion, we showed FASN expression in TNBC patients and Ki-67 proliferation index to be positively correlated with FASN level, indicating higher proliferation activity as FASN increases. However, there was no statistical association with PDL1 SP142, the currently FDA-approved assay, or FASN expression level.

\section{Ethics Statement}

The KUMC Institutional Review Board anonymized the archive data prior to the study and waived the requirement for prior consent (KUMC 202107-032).

\section{Availability of Data and Material}

The datasets generated or analyzed during the study are available from the corresponding author on reasonable request.

\section{Code Availability}

Not applicable.

\section{ORCID}

Jin Hee Park https://orcid.org/0000-0003-1277-7549

Hye Seung Han https://orcid.org/0000-0002-3591-9995

So Dug Lim https://orcid.org/0000-0003-2036-0313

Wook Youn Kim https://orcid.org/0000-0003-4024-8791

Kyoung Sik Park https://orcid.org/0000-0001-9806-9839

Young Bum Yoo https://orcid.org/0000-0003-0731-8807

Seung Eun Lee https://orcid.org/0000-0002-7459-0061

Wan-Seop Kim https://orcid.org/0000-0001-7704-5942

\section{Author Contributions}

Conceptualization: SEL, WSK. Data curation: KSP, YBY, JHP. Formal analysis: SEL, WYK. Funding acquisition: WSK. Investigation: JHP. Methodology: SEL. Supervision: WSK, HSH. Validation: SEL, SDL. Writing-original draft: JHP, SEL. Writing—review \& editing: SEL, WSK, JHP. Approval of final manuscript: all authors.

\section{Conflicts of Interest}

The authors declare that they have no potential conflicts of interest. 


\section{Funding Statement}

This research was supported by the Bio \& Medical Technology Development Program of the National Research Foundation (NRF) funded by the Korean government (Ministry of Science and ICT) (No. 2019M3E5D3073475).

\section{References}

1. Gluz O, Liedtke C, Gottschalk N, Pusztai L, Nitz U, Harbeck N. Triple-negative breast cancer: current status and future directions. Ann Oncol 2009; 20: 1913-27.

2. Diana A, Franzese E, Centonze S, et al. Triple-negative breast cancers: systematic review of the literature on molecular and clinical features with a focus on treatment with innovative drugs. Curr Oncol Rep 2018; 20: 76.

3. Lee KK, Kim JY, Jung JH, Park JY, Park HY. Clinicopathological feature and recurrence pattern of triple negative breast cancer. J Korean Surg Soc 2010; 79: 14-9.

4. Bianchini G, Balko JM, Mayer IA, Sanders ME, Gianni L. Triplenegative breast cancer: challenges and opportunities of a heterogeneous disease. Nat Rev Clin Oncol 2016; 13: 674-90.

5. Marotti JD, de Abreu FB, Wells WA, Tsongalis GJ. Triple-negative breast cancer: next-generation sequencing for target identification. Am J Pathol 2017; 187: 2133-8.

6. Lee SE, Park HY, Lim SD, Han HS, Yoo YB, Kim WS. Concordance of programmed death-ligand 1 expression between SP142 and 22C3/ SP263 assays in triple-negative breast cancer. J Breast Cancer 2020; 23: 303-13.

7. Rugo HS, Loi S, Adams S, et al. PD-L1 immunohistochemistry assay comparison in atezolizumab plus nab-paclitaxel-treated advanced triple-negative breast cancer. J Natl Cancer Inst 2021; 113: 1733-43.

8. Sun X, Wang M, Wang M, et al. Metabolic reprogramming in triple-negative breast cancer. Front Oncol 2020; 10: 428.

9. Gong Y, Ji P, Yang YS, et al. Metabolic-pathway-based subtyping of triple-negative breast cancer reveals potential therapeutic targets. Cell Metab 2021; 33: 51-64.

10. Cha YJ, Kim HM, Koo JS. Expression of lipid metabolism-related proteins differs between invasive lobular carcinoma and invasive ductal carcinoma. Int J Mol Sci 2017; 18: 232.

11. Sebastiani V, Visca P, Botti C, et al. Fatty acid synthase is a marker of increased risk of recurrence in endometrial carcinoma. Gynecol Oncol 2004; 92: 101-5.

12. Wang Y, Kuhajda FP, Li JN, et al. Fatty acid synthase (FAS) expression in human breast cancer cell culture supernatants and in breast cancer patients. Cancer Lett 2001; 167: 99-104.

13. Yang Y, Morin PJ, Han WF, et al. Regulation of fatty acid synthase expression in breast cancer by sterol regulatory element binding protein-1c. Exp Cell Res 2003; 282: 132-7.

14. Kusakabe T, Nashimoto A, Honma K, Suzuki T. Fatty acid synthase is highly expressed in carcinoma, adenoma and in regenerative epithelium and intestinal metaplasia of the stomach. Histopathology 2002; 40: 71-9.

15. Giro-Perafita A, Sarrats A, Perez-Bueno F, et al. Fatty acid synthase expression and its association with clinico-histopathological features in triple-negative breast cancer. Oncotarget 2017; 8: 74391-405.

16. Jiang W, Xing XL, Zhang C, et al. MET and FASN as prognostic biomarkers of triple negative breast cancer: a systematic evidence landscape of clinical study. Front Oncol 2021; 11: 604801.

17. Flores FH, Vinas G, Oliveras G, et al. Triple negative breast cancer: clinicopathologic characteristics and fatty acid synthase (FASN) ex- pression as a potential target. Ann Oncol 2014; 25(Suppl 4): IV60.

18. Goldhirsch A, Wood WC, Coates AS, et al. Strategies for subtypes: dealing with the diversity of breast cancer: highlights of the St. Gallen International Expert Consensus on the Primary Therapy of Early Breast Cancer 2011. Ann Oncol 2011; 22: 1736-47.

19. Hammond ME, Hayes DF, Dowsett M, et al. American Society of Clinical Oncology/College of American Pathologists guideline recommendations for immunohistochemical testing of estrogen and progesterone receptors in breast cancer (unabridged version). Arch Pathol Lab Med 2010; 134: e48-72.

20. Wolff AC, Hammond ME, Schwartz JN, et al. American Society of Clinical Oncology/College of American Pathologists guideline recommendations for human epidermal growth factor receptor 2 testing in breast cancer. Arch Pathol Lab Med 2007; 131: 18-43.

21. Sarrio D, Rodriguez-Pinilla SM, Hardisson D, Cano A, MorenoBueno G, Palacios J. Epithelial-mesenchymal transition in breast cancer relates to the basal-like phenotype. Cancer Res 2008; 68: 989-97.

22. Ma D, Jiang YZ, Xiao Y, et al. Integrated molecular profiling of young and elderly patients with triple-negative breast cancer indicates different biological bases and clinical management strategies. Cancer 2020; 126: 3209-18.

23. Li J, Holm J, Bergh J, et al. Breast cancer genetic risk profile is differentially associated with interval and screen-detected breast cancers. Ann Oncol 2015; 26: 517-22.

24. Dietze EC, Sistrunk C, Miranda-Carboni G, O’Regan R, Seewaldt VL. Triple-negative breast cancer in African-American women: disparities versus biology. Nat Rev Cancer 2015; 15: 248-54.

25. Foulkes WD, Smith IE, Reis-Filho JS. Triple-negative breast cancer. N Engl J Med 2010; 363: 1938-48.

26. Burstein MD, Tsimelzon A, Poage GM, et al. Comprehensive genomic analysis identifies novel subtypes and targets of triple-negative breast cancer. Clin Cancer Res 2015; 21: 1688-98.

27. Garrido-Castro AC, Lin NU, Polyak K. Insights into molecular classifications of triple-negative breast cancer: improving patient selection for treatment. Cancer Discov 2019; 9: 176-98.

28. D’Ippolito E, Iorio MV. MicroRNAs and triple negative breast cancer. Int J Mol Sci 2013; 14: 22202-20.

29. Le Du F, Eckhardt BL, Lim B, et al. Is the future of personalized therapy in triple-negative breast cancer based on molecular subtype? Oncotarget 2015; 6: 12890-908.

30. Hirai H, Tada Y, Nakaguro M, et al. The clinicopathological significance of the adipophilin and fatty acid synthase expression in salivary duct carcinoma. Virchows Arch 2020; 477: 291-9.

31. Kim S, Lee Y, Koo JS. Differential expression of lipid metabolismrelated proteins in different breast cancer subtypes. PLoS One 2015; 10: $\mathrm{e} 0119473$.

32. Giro-Perafita A, Rabionet M, Planas M, et al. EGCG-derivative G28 shows high efficacy inhibiting the mammosphere-forming capacity of sensitive and resistant TNBC models. Molecules 2019; 24: 1027.

33. Wang W, Bai L, Li W, Cui J. The lipid metabolic landscape of cancers and new therapeutic perspectives. Front Oncol 2020; 10: 605154.

34. Mrklic I, Capkun V, Pogorelic Z, Tomic S. Prognostic value of Ki67 proliferating index in triple negative breast carcinomas. Pathol Res Pract 2013; 209: 296-301.

35. Ahmed ST, Ahmed AM, Musa DH, Sulayvani FK, Al-Khyatt M, Pity IS. Proliferative index (Ki67) for prediction in breast duct car- 
cinomas. Asian Pac J Cancer Prev 2018; 19: 955-9.

36. Miyashita M, Ishida T, Ishida K, et al. Histopathological subclassification of triple negative breast cancer using prognostic scoring system: five variables as candidates. Virchows Arch 2011; 458: 65-72.

37. Nielsen TO, Leung SC, Rimm DL, et al. Assessment of Ki67 in breast cancer: updated recommendations from the international Ki67 in breast cancer working group. J Natl Cancer Inst 2021; 113: 808-19.

38. Zong Y, Zhu L, Wu J, et al. Progesterone receptor status and Ki-67 index may predict early relapse in luminal B/HER2 negative breast cancer patients: a retrospective study. PLoS One 2014; 9: e95629.

39. Wang W, Wu J, Zhang P, et al. Prognostic and predictive value of Ki-67 in triple-negative breast cancer. Oncotarget 2016; 7: 3107987.

40. Pan Y, Yuan Y, Liu G, Wei Y. P53 and Ki-67 as prognostic markers in triple-negative breast cancer patients. PLoS One 2017; 12: e0172324.

41. Mavratzas A, Seitz J, Smetanay K, Schneeweiss A, Jager D, Fremd C.
Atezolizumab for use in PD-L1-positive unresectable, locally advanced or metastatic triple-negative breast cancer. Future Oncol 2020; 16: 4439-53.

42. Dong H, Strome SE, Salomao DR, et al. Tumor-associated B7-H1 promotes T-cell apoptosis: a potential mechanism of immune evasion. Nat Med 2002; 8: 793-800.

43. Peg V, Lopez-Garcia MA, Comerma L, et al. PD-L1 testing based on the SP142 antibody in metastatic triple-negative breast cancer: summary of an expert round-table discussion. Future Oncol 2021; 17: 1209-18.

44. Li Y, Vennapusa B, Chang CW, et al. Prevalence study of PD-L1 SP142 assay in metastatic triple-negative breast cancer. Appl Immunohistochem Mol Morphol 2021; 29: 258-64.

45. Al-Jussani GN, Dabbagh TZ, Al-Rimawi D, Sughayer MA. Expression of PD-L1 using SP142 CDx in triple negative breast cancer. Ann Diagn Pathol 2021; 51: 151703. 